\title{
Universal effectiveness of high-depth circuits in variational eigenproblems
}

\author{
Joonho Kim $\odot,{ }^{1, *}, \dagger$ Jaedeok Kim $\odot,{ }^{2, *}$ and Dario Rosa $\oplus^{3,4, *}$ \\ ${ }^{1}$ School of Natural Sciences, Institute for Advanced Study, Princeton, New Jersey 08540, USA \\ ${ }^{2}$ AI Center, Samsung Research, Seoul 06765, Republic of Korea \\ ${ }^{3}$ School of Physics, Korea Institute for Advanced Study, 85 Hoegi-ro, Dongdaemun-gu, Seoul 02455, Republic of Korea \\ ${ }^{4}$ Department of Physics, Korea Advanced Institute of Science and Technology, 291 Daehak-ro, Yuseong-gu, Daejeon 34141, Republic of Korea
}

(Received 8 March 2021; accepted 7 May 2021; published 11 June 2021)

\begin{abstract}
We explore the effectiveness of variational quantum circuits in simulating the ground states of quantum manybody Hamiltonians. We show that generic high-depth circuits, performing a sequence of layer unitaries of the same form, can accurately approximate the desired states. We demonstrate their universal success by using two Hamiltonian systems with very different properties: the transverse field Ising model and the Sachdev-Ye-Kitaev model. The energy landscape of the high-depth circuits has a proper structure for the gradient-based optimization, i.e., the presence of local extrema—near any random initial points—reaching the ground level energy. We further test the circuit's capability of replicating random quantum states by minimizing the Euclidean distance.
\end{abstract}

DOI: 10.1103/PhysRevResearch.3.023203

\section{INTRODUCTION}

The variational quantum eigensolver (VQE) [1,2] is one of the most promising hybrid quantum-classical (HQC) algorithms, which may offer a precise approximation of the ground state of quantum systems. It is based on the iterative application of the following three steps: state preparation, measurement, and optimization. Let us briefly describe each step. First, the preparation of a trial state $|\psi(\boldsymbol{\theta})\rangle$ is carried out by successive application of unitary quantum gates that depend on variational parameters $\boldsymbol{\theta}$. Second, the measurement step estimates the trial state mean energy,

$$
E(\boldsymbol{\theta}) \equiv\langle\psi(\boldsymbol{\theta})|\mathcal{H}| \psi(\boldsymbol{\theta})\rangle,
$$

by taking the expectation value of the Hamiltonian $\mathcal{H}$ of the target system over the trial state. Third, the optimization step adjusts the variational parameters $\boldsymbol{\theta}$ of the trial wave function to minimize the mean state energy $E(\boldsymbol{\theta})$ by applying a classical optimization algorithm. See Ref. [3] and references therein for more details. After a sufficient number of iterations, the variational state $\left|\psi\left(\boldsymbol{\theta}^{*}\right)\right\rangle$ at a convergence point $\boldsymbol{\theta}=\boldsymbol{\theta}^{*}$ is expected to reproduce the ground state of the target Hamiltonian $\mathcal{H}$ well, under the assumption that the state Ansatz $|\psi(\boldsymbol{\theta})\rangle$ is parametrically expressible and well trainable under the gradient-based optimization. It is then a crucial question to find such an Ansatz.

Ideally, we are looking for a universally effective Ansatz capable of solving the VQE problem associated with an

\footnotetext{
*These authors contributed equally to this work.

†Corresponding author: joonhokim@ias.edu
}

Published by the American Physical Society under the terms of the Creative Commons Attribution 4.0 International license. Further distribution of this work must maintain attribution to the author(s) and the published article's title, journal citation, and DOI. arbitrary target Hamiltonian. Note that it is generically a very challenging task: Unless the target Hamiltonian is local, the ground states of nonlocal interacting systems are relatively close to typical quantum states that constitute most of the Hilbert space. Universality is thus equivalent to demanding that a circuit Ansatz approximate any random state $|\phi\rangle$ at certain values of the circuit parameters.

The existence of a parameter set $\varphi$, such that $|\psi(\varphi)\rangle \simeq|\phi\rangle$, is therefore a necessary condition for the effectiveness of the variational circuit. On top of that, we must be concerned with whether the gradient descent optimization can actually reach the parameters $\varphi$ from randomly initialized ones. Suppose that we use a layered quantum circuit composed of repetitive application of variational layers with the same architecture. Since increasing the number of layers extends the dimension of the parameter space, it can affect the circuit's expressibility only positively. However, there has been a reported tension between increasing depth of layers and trainability of the circuit via minimizing the mean energy, Eq. (1), known as the barren plateau phenomenon [4].

When the layered circuit reaches a certain depth such that it evolves to an approximate 2-design, a numerical experiment [4] has shown the exponential decay of the variance of energy gradients $\nabla_{\theta} E(\boldsymbol{\theta})$ with respect to the number $n$ of qubits-for random circuit states obtained by uniformly sampling from the parameter space. Combined with another fact, namely, that the random energy gradient vanishes on average, Chebyshev's inequality implies that the initial energy gradient can exponentially rarely deviate from zero. Such diminishing gradients hinder the beginning of efficient energy minimization, possibly causing the variational state to be stuck on nonoptimal plateaus.

There have been several proposals to overcome the vanishing gradient problem and optimize the variational circuit. The most obvious approach is to incorporate some physics information on the target Hamiltonian [5-8], e.g., 
the ground-state symmetry, for designing a less generic problem-tailored Ansatz. As an alternative direction towards the universal applicability of the variational algorithms, novel initialization [9,10], architecture [11,12], and optimization [13-15] of generic purpose circuits have been developed to enhance the circuit performance.

The main goal of this paper is to demonstrate the inherent capability of variational circuits as universal and accurate eigensolvers for generic Hamiltonians-if they can even approximate close-to-random states. To this end, we will simply put aside the barren plateau problem by sufficiently increasing the classical computation capacity. Specifically, we will consider the high-depth regime of the layered quantum circuit, thus building an exponentially-high-dimensional parameter space. In this regime, the variational circuit has sufficiently many layers and consequently many parameters. Hence the norm of the gradient vector $\left\|\nabla_{\theta} E(\boldsymbol{\theta})\right\|$ can still grow to a finite size, capable of moving the circuit parameter $\boldsymbol{\theta}$ from initial points, even though the magnitudes of the individual components $\left|\partial_{i} E(\boldsymbol{\theta})\right|$ are exponentially suppressed for the number of qubits $n$. We will illustrate the effectiveness of the high-depth circuits by solving two concrete VQE problems for the following quantum many-body Hamiltonians: the one-dimensional (1D) Ising model in a constant transverse magnetic field, which is a prototypical model of locally interacting spin-chain systems, and the Sachdev-Ye-Kitaev (SYK) model, which is a strongly interacting quantum mechanical system of Majorana fermions [16-18]. Despite the striking contrast in these two Hamiltonians' ground-state properties, we will find that the high-depth circuit achieves a very high fidelity in approximating both ground states. Moreover, we will see that the high-depth circuits can narrow the Euclidean distance from random states up to arbitrary precision, indicating outstanding expressibility and trainability that stems from the high dimensionality of $\boldsymbol{\theta}$.

The remarkable efficiency of the gradient descent optimization in the overparametrized regime was also reported for approximating random unitary matrices [19] and ground states [8] with certain variational Ansatze. More generally, overparametrization is an active research topic that underlies the success of deep learning. It makes large neural networks capable of reaching a global minimum during the optimization process, despite the nonconvexity of the energy landscape $[20,21]$. While searching for the ground state via the energy minimization, we will observe some phenomena similar to what happens during the training of the neural networks with large parameter spaces, summarized as follows:

First, the energy landscape looks fairly simple in the local vicinity of randomly chosen points [15]. Generically, an initial point is already confined in a certain basin of attraction, such that an emanating optimization trajectory can quickly arrive at a nearby local extremum. Especially if the circuit has enough layers to become an approximate 2-design, almost all uniformly sampled initial states end up with rather homogeneous energy levels.

Second, for high-depth circuits, all the local extrema in the energy landscape, reachable by the VQE optimization from randomly initialized points, are substantially close to the exact ground energy, i.e., the value of the global minimum. These minima are not isolated individual points but develop multiple (a)

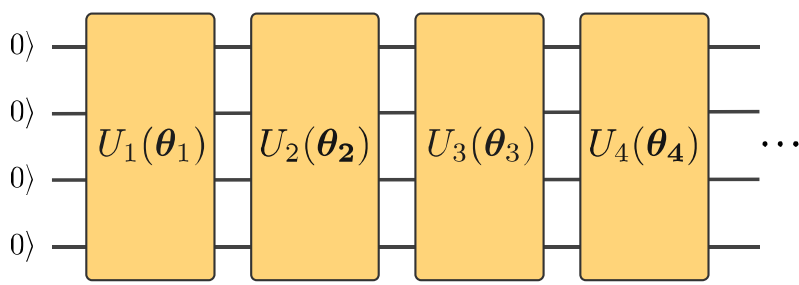

(b)

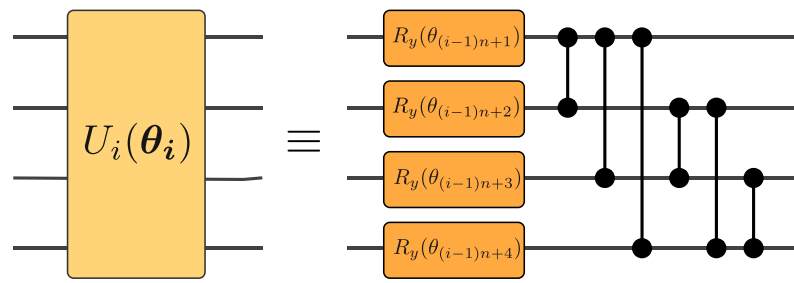

FIG. 1. The layered circuit Ansatz $|\psi(\boldsymbol{\theta})\rangle$ used in this paper (a) Circuit Ansatz. (b) Layer.

flat directions. This explains the robust success of the highdepth circuits in solving the VQE problems.

The rest of this paper is organized as follows. Section II introduces the architecture of the variational circuits used throughout this paper. Section III first reviews the occurrence of the barren plateau phenomenon and then examines the optimization of the variational circuits using the VQE example of the 1D Ising model coupled to a uniform transverse magnetic field. Section IV studies the same VQE problem for the SYK model, thus showing the universal effectiveness of the high-depth circuit. Section V addresses the ability of the variational circuits to reproduce typical states by minimizing the Euclidean distance. In particular, it shows that the highdepth Ansatz is highly expressible and trainable, being able to reach any random state. Finally, Sec. VI concludes with a discussion.

\section{CIRCUIT ANSATZE}

Our focus in this paper is to demonstrate the efficiency of high-depth layered circuits in a typical VQE problem, i.e., to approximate the ground state of a given Hamiltonian. To this purpose, here we specify the architecture of the variational circuit used in our numerical experiments. Our circuit state $|\psi(\theta)\rangle$ is composed of $L$ unitary layers acting sequentially on the initial product state $|0\rangle$, i.e.,

$$
|\psi(\boldsymbol{\theta})\rangle=U_{L}\left(\boldsymbol{\theta}_{L}\right) U_{L-1}\left(\boldsymbol{\theta}_{L-1}\right) \cdots U_{2}\left(\boldsymbol{\theta}_{2}\right) U_{1}\left(\boldsymbol{\theta}_{1}\right)|0\rangle,
$$

where each layer $U_{i}\left(\boldsymbol{\theta}_{i}\right)$ has single-qubit $y$-rotation $(R Y)$ gates, parametrized by $n$ periodic variables $\boldsymbol{\theta}_{i}$, and controlled- $z(C Z)$ gates operating on all pairs of $n$ qubits. More precisely, once the single-qubit $R Y$ gates have acted upon all individual qubits, the entangling $C Z$ gates acting on the $a$ th controlling and $b$ th targeting qubits are arranged for every integer pair $(a, b)$ satisfying $1 \leqslant a<b \leqslant n$. We have drawn the circuit state (2) with $n=4$ qubits in Fig. 1.

Note that we have deliberately chosen an unbiased circuit architecture, instead of embedding known properties of the ground states that are under investigation. Nonetheless, as we will see in Secs. III-V, the above circuit can solve the ground 
states of distinct Hamiltonians by minimizing $E(\boldsymbol{\theta})$ and accurately approximate random quantum states by minimizing the Euclidean distance, as long as the number of layers $L$ is sufficiently large.

\section{LOOKING INTO VQE TRAJECTORIES}

This section is devoted to the detailed investigation of the VQE optimization procedure, with a particular focus on the effectiveness of the high-depth circuit Ansatz (2). Our exploration will be based on a concrete Hamiltonian system, commonly used in measuring the performance of variational circuits, i.e., the 1D Ising model in a transverse and uniform magnetic field $[8,22]$.

The 1D transverse field Ising model is defined over a spin lattice of length $n$, consisting of the spin-spin coupling between nearest neighbors in the $z$ direction, as well as the spin interaction with a background uniform magnetic field along the transverse $x$ direction. Assuming periodic boundary conditions, $\sigma_{n+1}^{z} \equiv \sigma_{1}^{z}$, the ferromagnetic Ising Hamiltonian reads

$$
\mathcal{H} \equiv-\sum_{i=1}^{n} \sigma_{i}^{z} \sigma_{i+1}^{z}-g \sum_{i} \sigma_{i}^{x},
$$

where $\sigma_{i}^{x, y, z}$ is the Pauli operator acting on the $i$ th spin and $g$ denotes the strength of the uniform magnetic field.

The physics of this model has been well studied [23]. When the lattice size scales up to infinity, $n \rightarrow \infty$, the system undergoes a quantum phase transition at $|g|=1$ between the ordered $(|g|<1)$ and disordered $(|g|>1)$ phases. The former phase has the spin-flip $\mathbb{Z}_{2}$ symmetry that connects the two opposite ferromagnetic ground states, while the latter phase has a unique ground state, with all the spins aligned along the $x$ direction.

We will apply the VQE algorithm to the finite- $n$ Ising system, which exhibits some differences from the thermodynamic limit. Specifically, the $\mathbb{Z}_{2}$ degeneracy in the $0<|g|<1$ phase is broken at finite $n$. Our target state will be always nondegenerate and gapped at $|g| \neq 0$. All concrete calculations presented in this section have been done for $g=2$.

\section{A. Barren plateaus and classical resolution}

It was argued in Ref. [4] that optimization of the quantum variational circuits under the random parameter initialization comes with an inherent difficulty, given by the problem of vanishing gradients. For the circuit unitary ensemble that is quantum 2-design, random initial parameters are typically located on a plateau in the energy landscape. This means that the gradient-based optimization cannot even roll out. The odds of having nonvanishing gradients at random points decays exponentially with the system size $n$, impeding a large-scale application of the variational circuits. Its consequence would be even more detrimental on actual quantum devices, where the sampling noise must be taken into account $[4,14]$. Given its prominence, we begin by reviewing the vanishing gradient problem in the VQE setting.

Consider the ensemble of the energy gradients over the parameter space of the variational circuit in Fig. 1. To analyze the $k$ th component, $\partial_{k} E(\boldsymbol{\theta})$, that belongs to the $\ell$ th variational layer, it is convenient to group the $L$-layer unitaries (2) into the following two blocks:

$$
|\psi(\boldsymbol{\theta})\rangle=U_{-}\left(\boldsymbol{\theta}_{-}\right) U_{+}\left(\boldsymbol{\theta}_{+}\right)|0\rangle,
$$

where $\boldsymbol{\theta}_{q}=\left\{\theta_{p} \mid\left\lfloor\frac{p}{n}\right\rfloor=q\right\}, \boldsymbol{\theta}_{+}=\bigcup_{a \leqslant \ell} \boldsymbol{\theta}_{a}, \boldsymbol{\theta}_{-}=\bigcup_{a>\ell} \boldsymbol{\theta}_{a}$,

$$
\begin{aligned}
& U_{+}\left(\boldsymbol{\theta}_{+}\right) \equiv U_{\ell}\left(\boldsymbol{\theta}_{\ell}\right) U_{\ell-1}\left(\boldsymbol{\theta}_{\ell-1}\right) \cdots U_{2}\left(\boldsymbol{\theta}_{2}\right) U_{1}\left(\boldsymbol{\theta}_{1}\right), \\
& U_{-}\left(\boldsymbol{\theta}_{-}\right) \equiv U_{L}\left(\boldsymbol{\theta}_{L}\right) \cdots U_{\ell+2}\left(\boldsymbol{\theta}_{\ell+2}\right) U_{\ell+1}\left(\boldsymbol{\theta}_{\ell+1}\right) .
\end{aligned}
$$

As the partial derivative $\partial_{k}$ acts only on $U_{\ell}\left(\boldsymbol{\theta}_{\ell}\right)$, the variance of the $k$ th gradient component, $\operatorname{Var}_{\theta}\left[\partial_{k} E(\boldsymbol{\theta})\right]$, is

$$
-\int \frac{d \boldsymbol{\theta}}{(2 \pi)^{n L}}\left\langle\psi(\boldsymbol{\theta})\left|\left[U_{-}\left(\boldsymbol{\theta}_{-}\right) V_{k} U_{-}\left(\boldsymbol{\theta}_{-}\right)^{\dagger}, \mathcal{H}\right]\right| \psi(\boldsymbol{\theta})\right\rangle^{2},
$$

where $V_{k}$ denotes the Pauli operator $\sigma^{y}$ acting on the $k$ th spin variable, such that $\operatorname{Tr}\left(V_{k}\right)=0$ and $\operatorname{Tr}\left(V_{k}^{2}\right)=2^{n}$.

If we assume the quantum 2-design property of $U_{+}\left(\boldsymbol{\theta}_{+}\right)$ and/or $U_{-}\left(\boldsymbol{\theta}_{-}\right)$, the above integral (6) can be replaced with the unitary matrix integral. In that case, the matrix integral can be handled exactly and be simplified to

$$
\frac{2 \operatorname{Tr}\left(\mathcal{H}^{2}\right)}{2^{2 n}} .
$$

Such simplification (7) happens when both $U_{ \pm}\left(\boldsymbol{\theta}_{ \pm}\right)$are 2designs. Instead, if the 2-design condition does not hold for either of $U_{ \pm}\left(\boldsymbol{\theta}_{ \pm}\right)$, we have the following expressions:

$$
-\frac{1}{2^{2 n}} \int \frac{d \boldsymbol{\theta}_{-}}{(2 \pi)^{n(L-\ell)}} \operatorname{Tr}\left(\left[V_{k}, U_{-}\left(\boldsymbol{\theta}_{-}\right)^{\dagger} \mathcal{H} U_{-}\left(\boldsymbol{\theta}_{-}\right)\right]^{2}\right)
$$

if only $U_{+}\left(\boldsymbol{\theta}_{+}\right)$is a 2-design while $U_{-}\left(\boldsymbol{\theta}_{-}\right)$is not, or

$$
-\frac{\operatorname{Tr}\left(\mathcal{H}^{2}\right)}{2^{2 n}} \int \frac{d \boldsymbol{\theta}_{+}}{(2 \pi)^{n \ell}} \operatorname{Tr}\left(\left[V_{k}, U_{+}\left(\boldsymbol{\theta}_{+}\right)^{\dagger} \rho U_{+}\left(\boldsymbol{\theta}_{+}\right)\right]^{2}\right),
$$

where $\rho=|0\rangle\langle 0|$, if not $U_{+}\left(\boldsymbol{\theta}_{+}\right)$but only $U_{-}\left(\boldsymbol{\theta}_{-}\right)$is a 2-design. Asymptotically in the system size $n$, the above expressions (7)-(9) are all bounded as

$$
0 \leqslant \operatorname{Var}_{\theta}\left[\partial_{k} E(\boldsymbol{\theta})\right] \leqslant \frac{4 \operatorname{Tr}\left(\mathcal{H}^{2}\right)}{2^{2 n}}
$$

where we find the upper bound by expanding the commutator inside the integral of (8) and (9) and then applying the following trace inequality that holds for two Hermitian matrices $A$, $B$ [24]:

$$
\left|\operatorname{Tr}(A B)^{2 m}\right| \leqslant \operatorname{Tr}\left(A^{2 m} B^{2 m}\right) \quad \text { for } m \in \mathbb{N} .
$$

To determine the scaling behavior of the upper bound (10) with respect to the system size $n$, we examine how $\operatorname{Tr}\left(\mathcal{H}^{2}\right)$ scales with $n$ by extrapolating the values obtained from exact diagonalization of the Hamiltonian (3) up to $n \leqslant 17$. The result is presented in Fig. 2. We see that the term $\operatorname{Tr}\left(\mathcal{H}^{2}\right)$ scales as $2^{n}$, such that the exponential factor in the denominator of the upper bound (10) cannot fully be balanced by $\operatorname{Tr}\left(\mathcal{H}^{2}\right)$. Then, inserting the upper bound (10) into Chebyshev's inequality, which states

$$
\operatorname{Pr}\left(\left|\partial_{k} E(\boldsymbol{\theta})\right|>\epsilon\right)<\frac{\operatorname{Var}_{\boldsymbol{\theta}}\left[\partial_{k} E(\boldsymbol{\theta})\right]}{\epsilon^{2}},
$$

with $\partial_{k} E(\boldsymbol{\theta})$ being a random variable of zero mean, the probability of having a nonzero and finite derivative is exponentially 


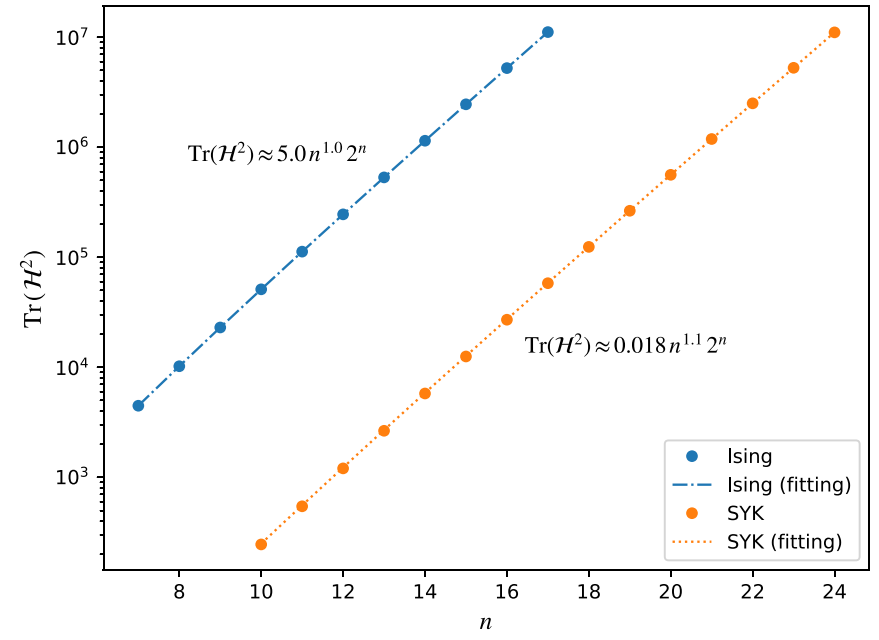

FIG. 2. $\operatorname{Tr}\left(\mathcal{H}^{2}\right)$ of the Ising model (3) and the SYK model (24) as a function of the system size $n$. The dashed lines are the regression lines with the functional form $a \cdot n^{b} 2^{n}$, based on the numerical data denoted as small circles.

suppressed with growing $n$. Therefore the large-scale VQE problems are expected to suffer from the vanishing gradients.

We have not yet justified the assumption that either of the unitaries $U_{ \pm}\left(\boldsymbol{\theta}_{ \pm}\right)$is quantum 2-design. To see if the problem of exponentially vanishing gradients happens in the circuit unitaries of Fig. 1, we numerically estimate the variance of initial gradients by collecting $10^{3}$ random energy gradients of the Ising model. Notice that it is much more efficient to use the parameter-shift rule for accurate estimation of the energy gradients [25]. Figure 3(a) clearly exhibits an exponential decay of the partial derivatives with the increasing number of qubits $n$, where the shaded region displays component-wise fluctuations of the variance for all $1 \leqslant k \leqslant n L$. This shows that the energy landscape for the circuit in Fig. 1 indeed contains the barren plateaus, which hinders the circuit optimization towards the ground state.

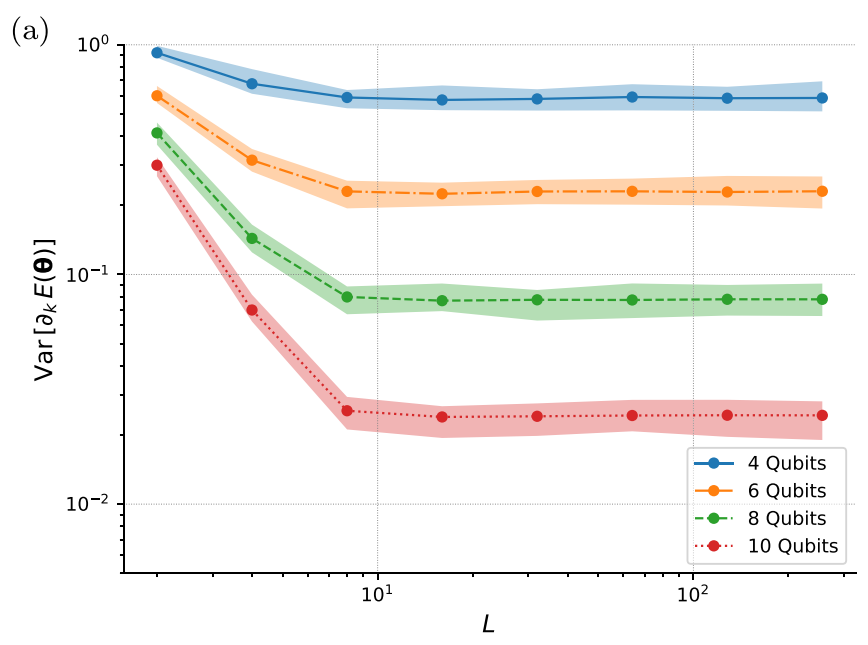

On the other hand, Fig. 3(a) shows that the variance, at a fixed value of $n$, converges to a constant by increasing the number of layers $L$. The variance is independent of $L$ beyond a transition point $L_{0}$, where the circuit unitaries with $L \geqslant L_{0}$ evolve to approximate 2-design. Due to the saturation, having exponentially many parameters can compensate for the exponential decay of individual components when calculating the gradient norm $\left\|\nabla_{\theta} E(\boldsymbol{\theta})\right\|$, which appears in the evolution of the circuit energy under the gradient descent with an infinitesimal rate $\alpha$ :

$$
\frac{d E(\boldsymbol{\theta})}{d \tau}=-\alpha\left\|\nabla_{\boldsymbol{\theta}} E(\boldsymbol{\theta})\right\|^{2} .
$$

Therefore the vanishing gradient problem inherent to the quantum Hilbert space can be resolved in a classical way, i.e., by using the exponentially-high-dimensional $\boldsymbol{\theta}$ space.

In agreement with the reasoning above, Fig. 3(b) exhibits a small initial drop dominated by the transient decrease in $\operatorname{Var}_{\theta}\left[\partial_{k} E(\boldsymbol{\theta})\right]$ for $L \leqslant L_{0}$ layers and then a steady increase driven by the linear growth in the number of circuit parameters. One can approximate the asymptotic increase rate of the norm $\left\|\nabla_{\theta} E(\boldsymbol{\theta})\right\|$ as

$$
\left\|\nabla_{\boldsymbol{\theta}} E(\boldsymbol{\theta})\right\| \sim \sqrt{n L \times \operatorname{Var}_{\theta}\left[\partial_{k} E(\boldsymbol{\theta})\right]},
$$

which agrees well with the numerically estimated growth rates between $\log \left\|\nabla_{\theta} E(\boldsymbol{\theta})\right\|$ and $\log L$ in Fig. 3(b)

$$
\begin{array}{ccccc}
n \text { qubits } & 4 & 6 & 8 & 10 \\
\hline \text { Rate } & 0.504 & 0.502 & 0.503 & 0.501
\end{array}
$$

We remark that the barren plateau phenomenon concerns only the initial steps of the gradient descent update. In almost all physical systems of relevance, the Hamiltonian spectrum is symmetrically distributed around a certain value, which we canonically set to zero. A random circuit state is therefore in a superposition of multiple Hamiltonian eigenstates, whose mean energy is almost certainly zero. On the other hand, the variational mean energy quickly becomes a negative value after a few steps of the VQE optimization, implying that the

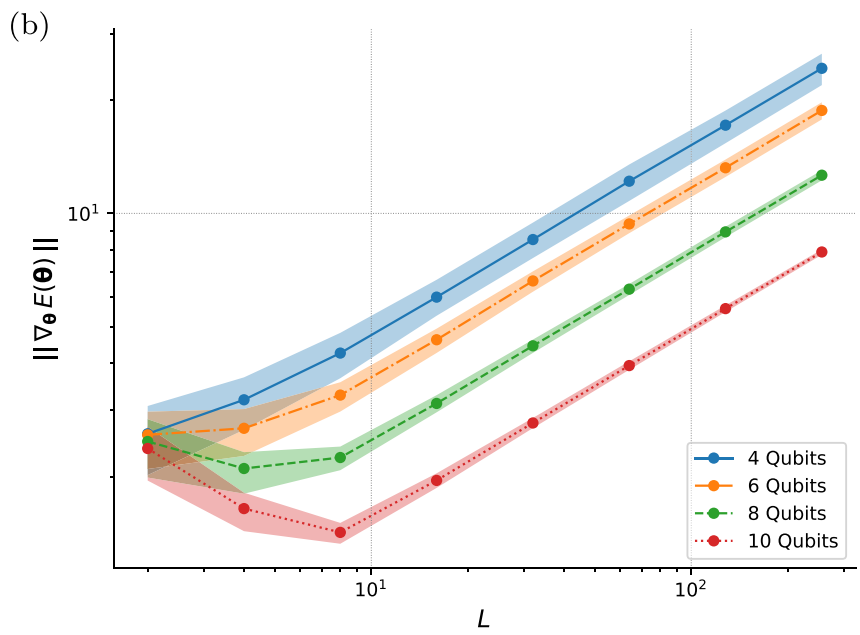

FIG. 3. The barren plateau experiment for the Ising Hamiltonian (3). (a) Sample variance of the energy derivative $\partial_{k} E(\boldsymbol{\theta})$. (b) Sample average of the Euclidean norm of $\nabla_{\boldsymbol{\theta}} E(\boldsymbol{\theta})$. The shaded areas indicate the variance across gradient components $\left\{\partial_{k} E(\boldsymbol{\theta})\right\}_{k=1}^{n L}$ in $(\mathrm{a})$ and the first and third sample quantiles in (b). 
circuit state is no longer represented by sample statistics of random circuit states.

Having found that the vanishing gradient problem can be trivially avoided at the cost of having exponentially many parameters, we turn to answering whether a variational circuit with sufficiently many layers can actually solve the VQE problems. We will examine the optimization error, the training curve, and the trajectory in the parameter space at different values of the circuit depth $L$.

\section{B. Optimizing the circuit}

We now come back to the original task of approximating the ground state of the Ising model, Eq. (3).

The VQE optimization results of the circuit states (2), with different numbers of layers $L$ and under the random initialization of the circuit parameters $\boldsymbol{\theta}$, can be summarized by the following two features:

(1) For small enough $L$, the minimized energies $E\left(\boldsymbol{\theta}^{*}\right)$ that the circuit states can reach are highly variable.

(2) For larger $L$, the $E\left(\boldsymbol{\theta}^{*}\right)$ distribution becomes concentrated around a mean value which gets smaller.

We illustrate these two features with the outcomes of the VQE algorithm for the case of a chain having $n=10$ sites and for $L \in\{8,10,12,14,16,18,20\}$ layers. To find the optimal parameters $\boldsymbol{\theta}^{*}$ that minimize the energy, we use the Adam optimization algorithm [26], which iteratively updates the variational parameters $\boldsymbol{\theta}$ by the exponential moving averages of gradients and their squares. It has a clear advantage in convergence speed, being widely used in a variety of deeplearning models. The parameter update rule is decided by a choice of three hyperparameters $\alpha, \beta_{1}, \beta_{2}$ as follows:

$$
\theta_{i, t+1}=\theta_{i, t}-\frac{\alpha m_{i, t}}{\sqrt{v_{i, t}}+\varepsilon},
$$

where

$$
m_{i, t}=\frac{\tilde{m}_{i, t}}{1-\beta_{1}^{t+1}}, \quad v_{i, t}=\frac{\tilde{v}_{i, t}}{1-\beta_{2}^{t+1}}
$$

and

$$
\begin{aligned}
\tilde{m}_{i, t} & =\beta_{1} \tilde{m}_{i, t-1}+\left(1-\beta_{1}\right) \partial_{i} E\left(\boldsymbol{\theta}_{t}\right), \\
\tilde{v}_{i, t} & =\beta_{2} \tilde{v}_{i, t-1}+\left(1-\beta_{2}\right)\left[\partial_{i} E\left(\boldsymbol{\theta}_{t}\right)\right]^{2} .
\end{aligned}
$$

$\beta_{1}$ and $\beta_{2}$ determine the weight ratio between the current gradient and the accumulated average of past gradients. Their values must be strictly larger than 0 and smaller than 1 . As is often the case for nonconvex optimization problems, there is no established method to find the best hyperparameters beyond trial-and-error approaches. We use the recommended values of $\beta_{1}$ and $\beta_{2}$ in Ref. [26], which perform well in various deep-learning applications.

$$
\begin{array}{ccc}
\alpha & \beta_{1} & \beta_{2} \\
\hline 0.05 & 0.9 & 0.999
\end{array}
$$

We have collected 35 independent VQE runs for the Ising Hamiltonian (3), whose initial parameters are randomly sampled from the uniform distribution $\mathcal{U}(0,2 \pi)^{\otimes n L}$, after 500 parameter updates. The sample distribution of the final VQE energy $E\left(\boldsymbol{\theta}^{*}\right)$ is visualized in Fig. 4 for different numbers of layers $L$. One can characterize it as follows. First, the

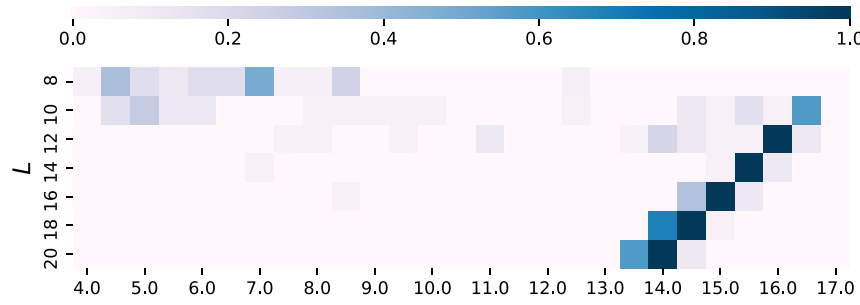

FIG. 4. Optimized VQE energy $\left[E\left(\boldsymbol{\theta}^{*}\right)-E_{0}\right]$ density for the Ising model over 35 distinct runs with random initialization.

energy distribution at the local extremum $\boldsymbol{\theta}^{*}$ clearly exhibits the widespread spectrum for a shallow depth, e.g.,

$$
\begin{array}{ll}
3.52 \leqslant E\left(\boldsymbol{\theta}^{*}\right)-E_{0} \leqslant 12.6 & \text { for } L=8, \\
4.67 \leqslant E\left(\boldsymbol{\theta}^{*}\right)-E_{0} \leqslant 16.9 & \text { for } L=10,
\end{array}
$$

sometimes achieving a relatively good energy level while the gap always persists. Second, by stacking more layers, i.e., $L \geqslant 12$, deeper than the 2-design transition point detected in Fig. 3(a), the energy distribution starts to concentrate around a value, which is far from the ground energy $E_{0}$. Third, the
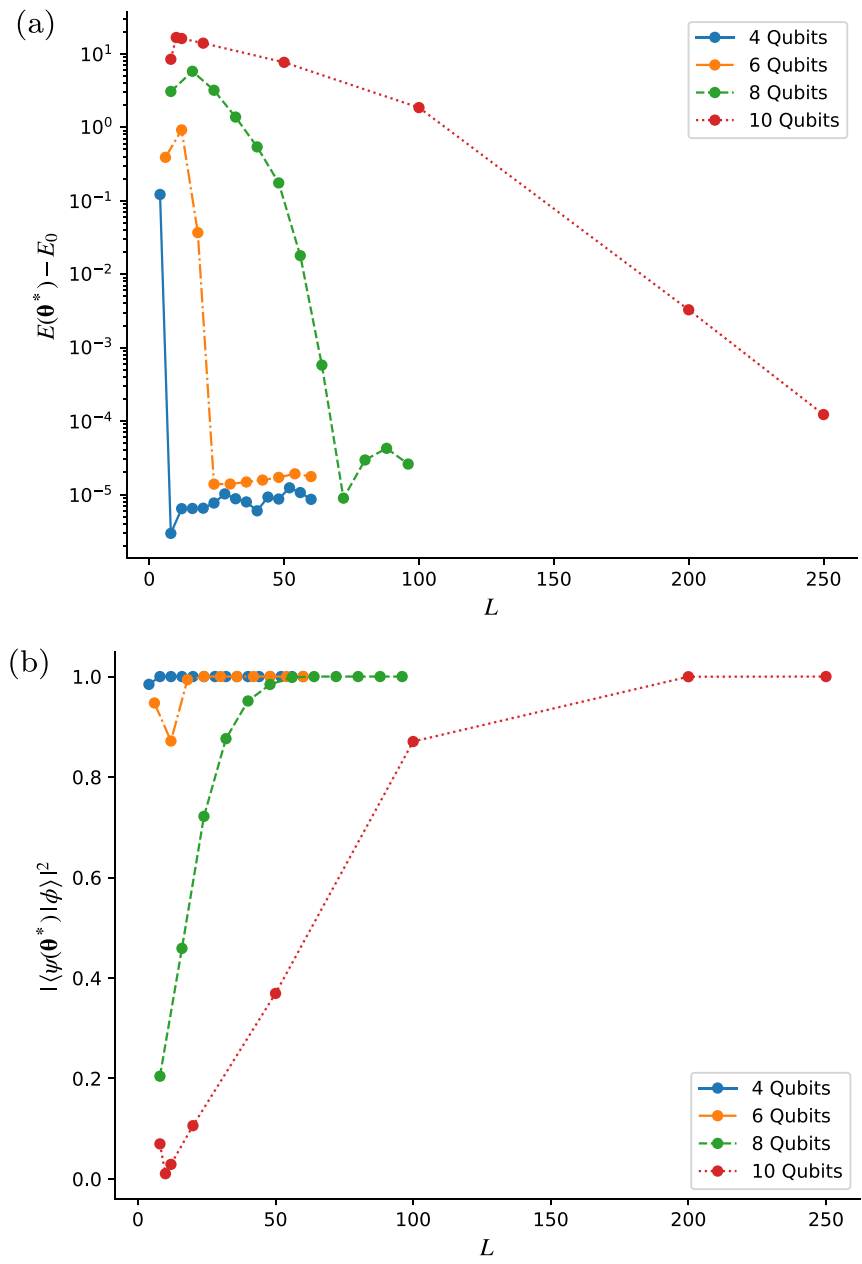

FIG. 5. Single-run VQE outcomes for the Ising model (3) using the layered circuit Ansatz (2) at different depths $L$. (a) The VQE optimization error $E\left(\boldsymbol{\theta}^{*}\right)-E_{0}$. (b) Fidelity between the optimized and true ground states. 

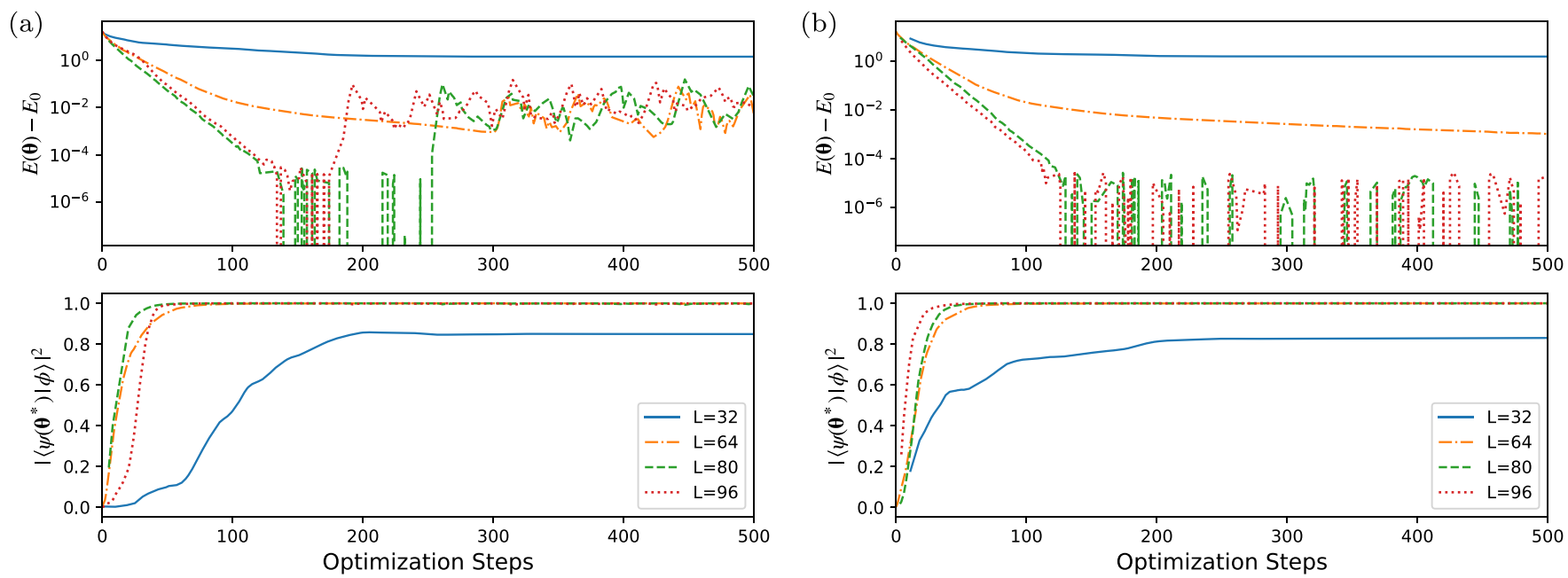

FIG. 6. Optimization curves of the circuit state on the Ising model at 8 qubits. The upper and lower plots denote the VQE error $E\left(\boldsymbol{\theta}_{\tau}\right)-E_{0}$ and the fidelity $\left|\left\langle\psi\left(\boldsymbol{\theta}^{*}\right) \mid \phi\right\rangle\right|^{2}$ between the circuit and true ground states, respectively, as a function of the optimization steps $\tau$. Notice that the late-time fluctuation has been alleviated with the learning rate scheduling. (a) With the constant hyperparameters. (b) With the exponential decay (21) of hyperparameters.

average value of $E\left(\boldsymbol{\theta}^{*}\right)$ continues to decrease for the growing number of layers $L$, suggesting that the high-depth variational circuits can possibly simulate the ground state using the VQE optimization.

Encouraged by the observed shrinkage of the mean and variance of $E\left(\boldsymbol{\theta}^{*}\right)$, we have also done the single VQE tryouts for a broader span of $(n, L)$, as summarized in Fig. 5. It is clear that being deeper enhances the variational circuit's capability to replicate the ground state $\left|\psi_{0}\right\rangle$, reaching the ground-state energy $E_{0}$ and achieving a high level of fidelity with $\left|\psi_{0}\right\rangle$. The high-depth circuits can achieve a zero error with the following precision:

$$
\left|E(\boldsymbol{\theta})-E_{0}\right| \leqslant 10^{-5} \times \Delta E,
$$

where $\Delta E$ denotes the bandwidth of the target Hamiltonian $\mathcal{H}$, defined as the difference between the largest and smallest eigenvalues of $\mathcal{H}$. We note that such precision can be achieved only with an appropriately chosen learning rate $\alpha$, in order to avoid too-large parameter updates that prevent the fine-level optimization. Here, we refrain from the systematic hyperparameter search, which may be more relevant for the case where the average gap between nearby energy levels shrinks, and simply stick to $\alpha=0.05(L=4,6,8)$ and $\alpha=0.01(L=$ 10). We have found that (19) can be achieved when the circuit depth $L$ passes through the threshold value

\begin{tabular}{ccccc}
$n$ qubits & 4 & 6 & 8 & 10 \\
\hline$L_{v}$ layers & 10 & 24 & 68 & 250
\end{tabular}

We also remark that deeper circuits do not necessarily lead to better performance with VQE, as displayed by the gentle ramp after passing the threshold point $L_{v}$. This can be understood as follows: As the space of variational parameters $\boldsymbol{\theta}$ has more dimensions, the basin of attraction to local extrema becomes narrower [11], giving a larger value of the estimated inverse volume [27]

$$
V_{k}^{-1}=\sum_{i=1}^{k} \ln \lambda_{i}(H),
$$

where the summation is taken over the top- $k$ eigenvalues $\left\{\lambda_{i}(H)\right\}_{i=1}^{k}$ of the Hessian matrix $H_{i j} \equiv \partial_{i} \partial_{j} E(\boldsymbol{\theta})$. [For instance, the positive correlation between $L$ and $V_{k}^{-1}(L)$ can clearly be identified in Fig. 7(a), drawn for $k=100$.] As a result, the VQE trajectory is unlikely to land at an exact extremum but is likely to wander around nearby points, whose deviation gets larger as the attractor basin becomes narrower and steeper.

Even with the optimal number of layers, such that the circuit state can reach an exact extremum and accurately represent the ground state $\left|\psi_{0}\right\rangle$ of the Ising Hamiltonian (3), the narrowness of the attractor basin still makes the VQE trajectory somewhat unstoppable, passing through the best point $\boldsymbol{\theta}^{*}$ and then hopping around in the local neighborhood. Figure 6(a) shows that the VQE error $E\left(\boldsymbol{\theta}_{\tau}\right)-E_{0}$ slightly increases on average and mildly fluctuates after achieving the minimum error $E\left(\boldsymbol{\theta}^{*}\right)-E_{0}$. This residual error can be reduced by making use of popular optimization tricks, such as early stopping or learning rate scheduling, which causes the VQE optimization to stop at the optimal point. For instance, by introducing the exponential decay of the learning rate $\alpha$, i.e.,

$$
\alpha_{\tau}=\alpha_{0} c^{\tau / 500}, \quad \tau \geqslant 0
$$

at the optimization step $\tau$ with a constant value $c=0.3$, we could reduce the late-time fluctuations as in Fig. 6(b).

So far, we have discussed some important aspects of the VQE optimization. The main observation is that, when supported by the high-dimensional parameter space, randomly initialized variational circuits can approximate the Ising ground state with remarkably high accuracy. The energy gradient neither vanishes nor randomly fluctuates along the optimization trajectory, making it quickly converge to a local minimum that exhibits a small energy gap from the ground energy $E_{0}$ and high fidelity with the exact ground state $\left|\psi_{0}\right\rangle$. We will explore this efficiency of the high-depth circuit in some detail by visualizing the VQE trajectory on the energy landscape. 


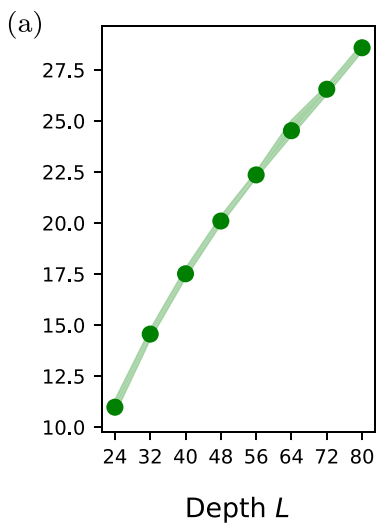

(b)
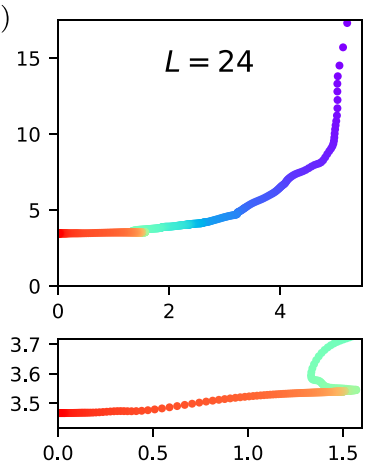
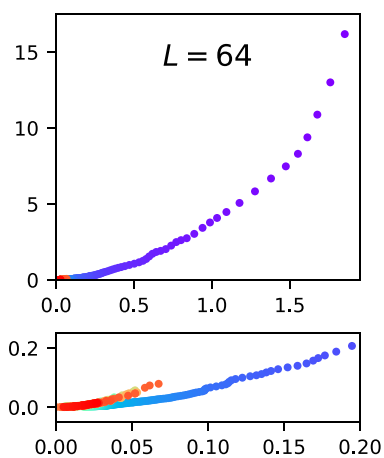

$S_{k}\left(\theta^{*}\right)$-projected distance

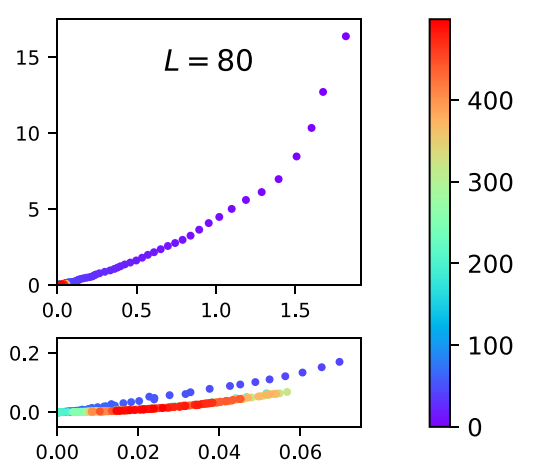

300

0

0

FIG. 7. The visualization of the optimization trajectory in the 100 steepest directions for the Ising model with $n=8$ qubits. (a) The mean of the top 100 Hessian eigenvalues. (b) The horizontal and vertical axes denote the $\mathcal{S}_{100}\left(\boldsymbol{\theta}^{*}\right)$-projected Euclidean distance and the VQE error $E\left(\boldsymbol{\theta}_{\tau}\right)-E_{0}$, respectively. The bottom boxes magnify the trajectory near the optimal point $\boldsymbol{\theta}^{*}$.

\section{Visualizing the trajectory}

A key characteristic that contributes to the success of the high-depth circuit is the fact that randomly initialized points are likely to be already confined in the basin of attraction of a good attractor, i.e., a local extremum that is close enough to the ground-state energy. This point will now be illustrated by looking at the actual optimization trajectories under the energy minimization.

For a given initial point $\boldsymbol{\theta}_{0}$ and the trajectory $\mathcal{T}\left(\boldsymbol{\theta}_{0}\right)=\left\{\boldsymbol{\theta}_{\tau}\right\}$ thereafter, we identify the optimal parameter $\boldsymbol{\theta}^{*}$ as the point in the trajectory $\mathcal{T}\left(\boldsymbol{\theta}_{0}\right)$ having the minimum energy expectation value

$$
E\left(\boldsymbol{\theta}^{*}\right) \leqslant E\left(\boldsymbol{\theta}_{\tau}\right) \text { for any } \tau \geqslant 0,
$$

which is the best possible representative point of the local extrema that the trajectory $\mathcal{T}\left(\boldsymbol{\theta}_{0}\right)$ converges around. The associated attractor basin can be estimated by calculating the Hessian $H_{i j} \equiv \partial_{i} \partial_{j} E\left(\boldsymbol{\theta}^{*}\right)$ at the optimum $\boldsymbol{\theta}^{*}$. We are interested in the eigenvalue spectrum, $\left\{\lambda_{i}(H)\right\}$, from which we distinguish steep and flat directions and calculate the degree of steepness.

Similar to the case of deep neural networks [27-29], the local extrema in the VQE energy landscape of high-depth circuits are often interconnected in multiple flat directions, whose corresponding Hessian eigenvalues are zero, looking like valleys rather than isolated singular points. The success of the VQE algorithm is not affected by a specific position in flat directions, but is only concerned with whether a ball, initially at a considerable height, can roll off in steep directions and reach a sufficiently deep gorge. This motivates us to consider the $k$-dimensional hypersurface $\mathcal{S}_{k}\left(\boldsymbol{\theta}^{*}\right)$ along the $k$ steepest directions, i.e., spanned by the top- $k$ Hessian eigenvectors. More precisely, we want to examine whether the $\mathcal{S}_{k}\left(\boldsymbol{\theta}^{*}\right)$-projected Euclidean distance between $\boldsymbol{\theta}^{*}$ and $\boldsymbol{\theta}_{\tau}$ decreases along the trajectory $\mathcal{T}\left(\boldsymbol{\theta}_{0}\right)$ as the optimization step $\tau$ progresses, until $E\left(\boldsymbol{\theta}_{\tau}\right)=E\left(\boldsymbol{\theta}^{*}\right)$. This will tell us whether the entire trajectory $\mathcal{T}\left(\boldsymbol{\theta}_{0}\right)$ is confined in the $k$-dimensional basin of attraction, while ignoring the movement in the directions of less or zero attraction.

Figure 7(b) displays the VQE error $E\left(\boldsymbol{\theta}_{\tau}\right)-E_{0}$ and the projection distance $\left\|\boldsymbol{\theta}_{\tau}-\boldsymbol{\theta}^{*}\right\|_{\mathcal{S}_{100}\left(\boldsymbol{\theta}^{*}\right)}$ along the actual optimization trajectories, whose step number $\tau$ is indicated by color. We have made the following observations: First, when an appropriate value of $k$ is selected, both the distance $\left\|\boldsymbol{\theta}_{\tau}-\boldsymbol{\theta}^{*}\right\|_{\mathcal{S}_{k}\left(\boldsymbol{\theta}^{*}\right)}$ and the loss value $E\left(\boldsymbol{\theta}_{\tau}\right)-E_{0}$ continuously decrease on a macroscopic scale. This exhibits that the trajectory $\mathcal{T}\left(\boldsymbol{\theta}_{0}\right)$ converges without escaping from a specific basin of the attractor that encloses a randomly initialized point $\boldsymbol{\theta}_{0}$. Second, for a shallow circuit state, the optimization trajectory often makes a slight detour in some orthogonal directions. In contrast, the steady convergence occurs typically for large $L$. This implies that the vicinity of any randomly initialized parameters is effectively convex. Finally, we find from Fig. 7(a) that the attractor basin in the direction of $\mathcal{S}_{k}\left(\boldsymbol{\theta}^{*}\right)$ evolves rapidly to be steeper and narrower as the depth $L$ increases, thereby causing the rapid convergence and substantial late-time fluctuation around $\boldsymbol{\theta}^{*}$. It is noticeable that the initial convergence follows a milder path than later fluctuations in the $L=64$ case, while the convergence in the $L=80$ case happens along a much steeper route. Taken together, these observations show the quick convergence of high-depth circuits [8,19] under the VQE algorithm.

\section{SOLVING THE SYK MODEL}

Our discussions so far have been based on just a particular Hamiltonian, Eq. (3), the Ising model in a transverse uniform magnetic field. Since the variational circuit that we use has no features particularly tailored to the Ising model, we expect it to closely replicate the ground states of other Hamiltonians in the high-depth regime. To check the generality of the prior discussions on the efficiency of the high-depth circuits, we will now solve the VQE problem, defined for another Hamiltonian of a very distinct nature: the Sachdev-Ye-Kitaev (SYK) model.

\section{A. The SYK Model}

The SYK model [16-18] is built out of $2 n$ Majorana fermions in $1 \mathrm{D}$, i.e., the operators $\gamma_{i}$, with $i=1, \ldots, 2 n$, satisfying the following anticommutation relations:

$$
\left\{\gamma_{i}, \gamma_{j}\right\}=\delta_{i j}
$$


where $\delta_{i j}$ denotes the Kronecker delta. The SYK Hamiltonian is an all-to-all Hamiltonian, which couples all the Majorana fermions together in a fully nonlocal fashion, consisting of the following $q$-body interaction terms with $q \geqslant 2$ being an even integer:

$$
\mathcal{H} \equiv(i)^{q / 2} \sum_{i_{1}<\cdots<i_{q}} J_{i_{1} \cdots i_{q}} \gamma_{i_{1}} \cdots \gamma_{i_{q}}
$$

where the coupling constants $J_{i_{1} \cdots i_{q}}$ are randomly sampled from the Gaussian distribution of mean 0 and variance

$$
\left\langle J_{i_{1} \cdots i_{q}}^{2}\right\rangle \equiv \frac{J^{2}(q-1) !}{(2 n)^{q-1}},
$$

where $J^{2}$ is a constant which we set to be equal to 1 . The model has recently attracted widespread attention from different communities, due to some peculiar features that it enjoys. It has been shown that when $q \geqslant 4$, the model is highly chaotic [17,18,30,31], although solvable in the large- $n$ limit $[17,18]$, thus creating a perfect situation to study relevant questions on quantum chaos which are usually out of reach for other chaotic models. Moreover, the SYK model has intriguing connections with the physics of black holes and quantum gravity, promoting itself as an ideal candidate to address new questions on holography and the anti-de Sitter and conformal field theory (AdS-CFT) correspondence.

We will focus our attention on the SYK model with $q=4$. It has two notable features that can be a source of trouble for any eigensolver algorithms, regardless of being classical or quantum mechanical, whose goal is to reach the ground state. First, the energy spectrum of the SYK model is very dense, especially having a small energy gap between the ground state $E_{0}$ and all other excited states. Second, the SYK ground state is much less distinguishable from generic quantum states in the Hilbert space, supporting the volume-law scaling of the entanglement entropy. Therefore the VQE computation with the SYK model must be seen as a highly challenging benchmark [32].

As another characteristic, the SYK model is known to have twofold degenerate eigenstates, if and only if $n=4 k+2$ for any positive integer $k[30,31]$. Denoting the twofold degenerate ground states by $\left|\phi_{1}\right\rangle$ and $\left|\phi_{2}\right\rangle$, which are orthonormal to each other, the VQE target states for the SYK model are then given by all the possible linear combinations of the form

$$
\left|\phi_{0}\right\rangle=\frac{\alpha}{\sqrt{|\alpha|^{2}+|\beta|^{2}}}\left|\phi_{1}\right\rangle+\frac{\beta}{\sqrt{|\alpha|^{2}+|\beta|^{2}}}\left|\phi_{2}\right\rangle,
$$

with $\alpha$ and $\beta$ being complex numbers. Therefore the distance between the circuit state $|\psi(\boldsymbol{\theta})\rangle$ and the closest state of the form $\left|\phi_{0}\right\rangle$ can be measured by computing

$$
\left|\left\langle\psi(\boldsymbol{\theta}) \mid \phi_{1}\right\rangle\right|^{2}+\left|\left\langle\psi(\boldsymbol{\theta}) \mid \phi_{2}\right\rangle\right|^{2} \leqslant 1
$$

with the inequality which is saturated whenever $|\psi(\boldsymbol{\theta})\rangle$ takes exactly the form (26).

We will numerically show that the high-depth quantum circuit can effectively learn the ground state of the SYK model. This will imply that even complicated systems, involving nonlocal interactions and a high level of entanglement, can be universally simulated through the variational circuits.

\section{B. Optimizing the circuit}

We start by discussing whether the SYK Hamiltonian causes the vanishing gradient problem for the variational circuit in Fig. 1. When at least one of $U_{ \pm}\left(\boldsymbol{\theta}_{ \pm}\right)$is a 2-design, the scaling behavior of $\operatorname{Tr}\left(\mathcal{H}^{2}\right)$ will determine whether the SYK energy gradient will be exponentially suppressed or not. The Hamiltonian (24) has been exactly diagonalized up to $n=15$. Moreover, an approximate analytical formula of the spectral density can be found in Ref. [33]; we use it to extrapolate $\operatorname{Tr}\left(\mathcal{H}^{2}\right)$ for the large system size $n$. The results are presented in Fig. 2. We clearly see, as in the Ising model, that $\operatorname{Tr}\left(\mathcal{H}^{2}\right)$ scales as $2^{n}$, which indicates the abundance of the barren plateaus in the VQE energy landscape of the SYK model.

The vanishing gradient problem has also been observed numerically by computing the sample variance of $\partial_{k} E(\boldsymbol{\theta})$ over a collection of 1000 random parameters, as displayed in Fig. 8(a). Clearly, the energy gradient $\partial_{k} E(\boldsymbol{\theta})$ is exponentially suppressed by increasing $n$. We also see that for the SYK model, contrary to the Ising Hamiltonian, there is almost no transient regime where $\partial_{k} E(\boldsymbol{\theta})$ is still large, yet decreasing for the growing number of layers $L$. The lack of the transient regime, which is a consequence of the nonlocal nature of the SYK interactions [11], is a clear obstacle in using the variational circuit to approximate the SYK ground state in the low-depth regime.

On the other hand, as we can see from Fig. 8(b), the norm of the gradient vector is again increasing for the growing number of layers $L$, due to the saturation of the $\operatorname{Var}_{\theta}\left[\partial_{k} E(\boldsymbol{\theta})\right]$ with respect to $L$. The empirically measured growth rates between $\ln \left\|\nabla_{\theta} E(\boldsymbol{\theta})\right\|$ and $\ln L$ are

\begin{tabular}{ccccc}
$n$ qubits & 4 & 6 & 8 & 10 \\
\hline Rate & 0.503 & 0.503 & 0.501 & 0.502
\end{tabular}

matching the simple estimation formula (14). This tells us that the gradient-based optimization can at least launch for highdepth quantum circuits, which bypass the vanishing gradient problem using an exponentially-high-dimensional parameter space.

As the next step, we examine the performance of the variational circuit Ansatz (2) in reaching the ground state of the SYK Hamiltonian, by repeating the same numerical experiments conducted in Sec. III B under the same choice of hyperparameters (18).

First, the sample distribution of the minimized energy $E\left(\theta^{*}\right)$ for randomly initialized circuits with $4 \leqslant L \leqslant 16$ layers is illustrated in Fig. 9, based on 35 sample VQE runs at each $L$. A notable observation is the small variance of the final energy, compared with the Ising VQE energy distribution in Fig. 4, even at very low depth. We interpret it as another manifestation of the fact that the ground state that we aim to approximate is less distinguishable from generic quantum states. Hence developing an optimization trajectory demands more classical computing power through the highdimensional $\boldsymbol{\theta}$ space [32]. Another-and perhaps the most important - point to stress is that the mean value of the minimized circuit energy $E\left(\boldsymbol{\theta}^{*}\right)$ decreases by stacking more layers, just like what has happened to the Ising VQE problem. This suggests that the high-depth circuits can reach a very good approximation of the SYK ground states. 

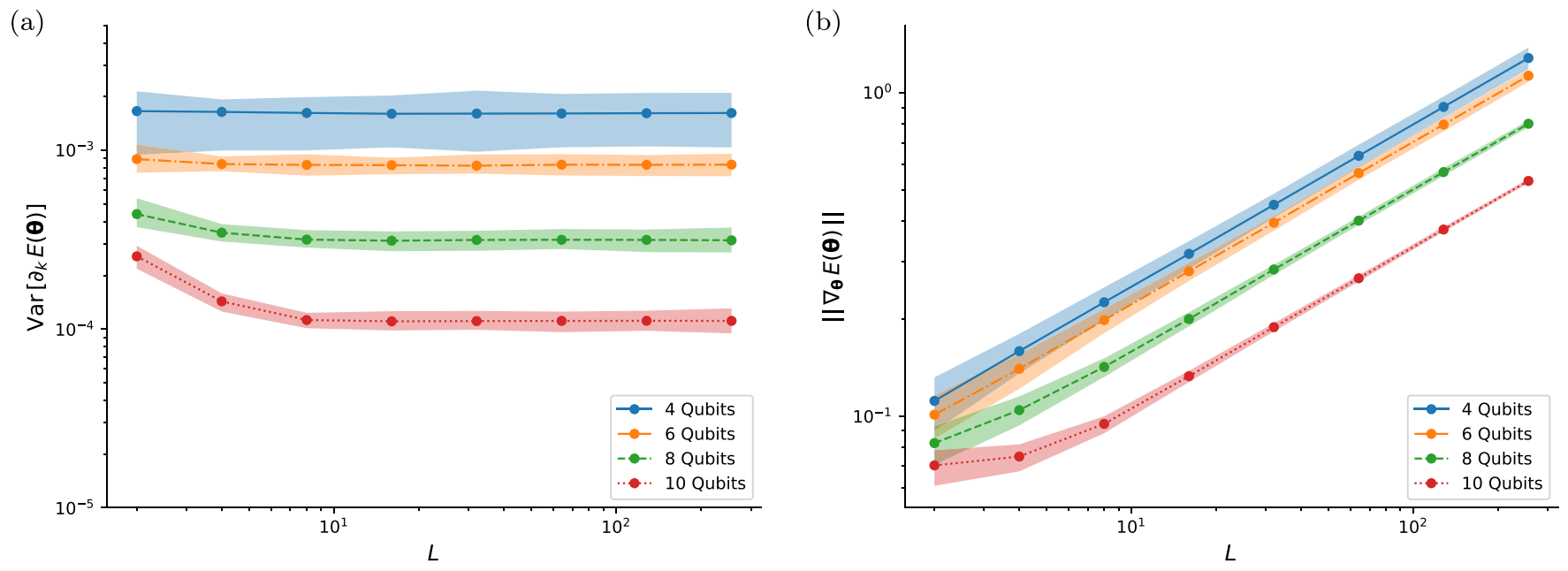

FIG. 8. The barren plateau experiment for the SYK Hamiltonian (24). (a) Sample variance of the energy derivative $\partial_{k} E(\boldsymbol{\theta})$. (b) Sample average of the Euclidean norm of $\nabla_{\boldsymbol{\theta}} E(\boldsymbol{\theta})$. The shaded areas indicate the variance across gradient components $\left\{\partial_{k} E(\boldsymbol{\theta})\right\}_{k=1}^{n L}$ in $($ a) and the first and third sample quantiles in (b).

Second, we have measured the performance of the layered circuit Ansatz (2) in approximating a ground state of the SYK model (24), as a function of the circuit depth. Specifically, the VQE single-run error $E\left(\boldsymbol{\theta}^{*}\right)-E_{0}$ is drawn in Fig. 10. The high-depth circuit performs very well, reaching a zero error with the following accuracy:

$$
\left|E(\boldsymbol{\theta})-E_{0}\right| \leqslant 10^{-5} \times \Delta E,
$$

when the depth $L$ arrives at the values

$$
\begin{array}{ccccc}
n \text { qubits } & 4 & 6 & 8 & 10 \\
\hline L_{v} \text { layers } & 12 & 30 & 96 & 220
\end{array}
$$

We also note from Fig. 10(b) that the fidelity between the optimized and ground states tends to decrease at an intermediate scale of depth, while the VQE error continues to be reduced without temporary increase. Such contrasting behavior is due to the dense energy spectrum of the SYK model near the ground energy level $E_{0}$. With an intermediate-depth circuit, the VQE algorithm is going to approximate not the exact ground states, but some low-lying excited states. In this way, the energy continues to decrease, but the fidelity does not improve. However, for sufficiently deep circuits, the VQE algorithm can overcome this difficulty and reach an excellent agreement with the ground state.

Interestingly, we have seen that the necessary numbers of layers, $L_{v}$, to reach the high precision (19) are roughly of the

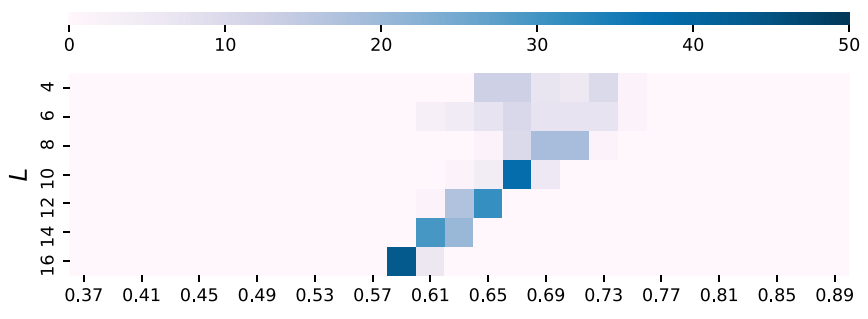

FIG. 9. Optimized VQE energy $\left[E\left(\boldsymbol{\theta}^{*}\right)-E_{0}\right]$ density for the $S Y K$ model over 35 distinct runs with random initialization. same order for both the SYK and Ising models. We will also see that the circuit (2) with $L \geqslant L_{v}$ achieves high precision in replicating random states by minimizing the Euclidean distance, as shown in Fig. 11. This compatibility highlights the universal effectiveness of the high-depth circuit in approximating any quantum state in the Hilbert space, both generic and nongeneric ones.

\section{APPROXIMATING RANDOM STATES USING THE EUCLIDEAN LOSS FUNCTION}

Taking one step further, we will look into the high-depth circuit's capability to approximate generic quantum states $|\phi\rangle$ by minimizing the Euclidean distance.

Recall that the variational circuit $|\psi(\boldsymbol{\theta})\rangle$ with $L$ layers depends on $n L$ parameters, which are periodic over the finite interval $[0,2 \pi)$ up to an overall sign. It is a mapping from the $n L$-dimensional torus to the Hilbert space of $n$ qubits. As a practical measure for the expressibility and trainability of the circuits in simulating quantum states, we consider whether, for a given quantum state $|\phi\rangle$, there exist a point $\boldsymbol{\theta}^{*}$ in the parameter space, reachable by the gradient-based optimization such that $\left|\psi\left(\theta^{*}\right)\right\rangle \simeq|\phi\rangle$. Such a measure of expressibility is motivated by Ref. [34] but tailored for the hybrid algorithms. Its definition follows:

Let us apply the gradient descent to find the minimum distance at the closest point

$$
\boldsymbol{\theta}^{*}=\arg \min _{\boldsymbol{\theta}} \||\psi(\boldsymbol{\theta})\rangle-|\phi\rangle \|
$$

between the circuit and target states, where $\|\cdot\|$ is the Euclidean norm of a complex vector. The (in)expressibility of the variational circuit is written as the average of the minimum distances over all quantum states:

$$
\varepsilon=\frac{1}{\operatorname{Vol}\left[U\left(2^{n}\right)\right]} \int d \mu_{\phi} \min _{\boldsymbol{\theta}} \||\psi(\boldsymbol{\theta})\rangle-|\phi\rangle \| .
$$

Notice that the closeness between two quantum states is usually defined by using the trace distance, which is highly sensitive to small parameter changes. Instead, we have 

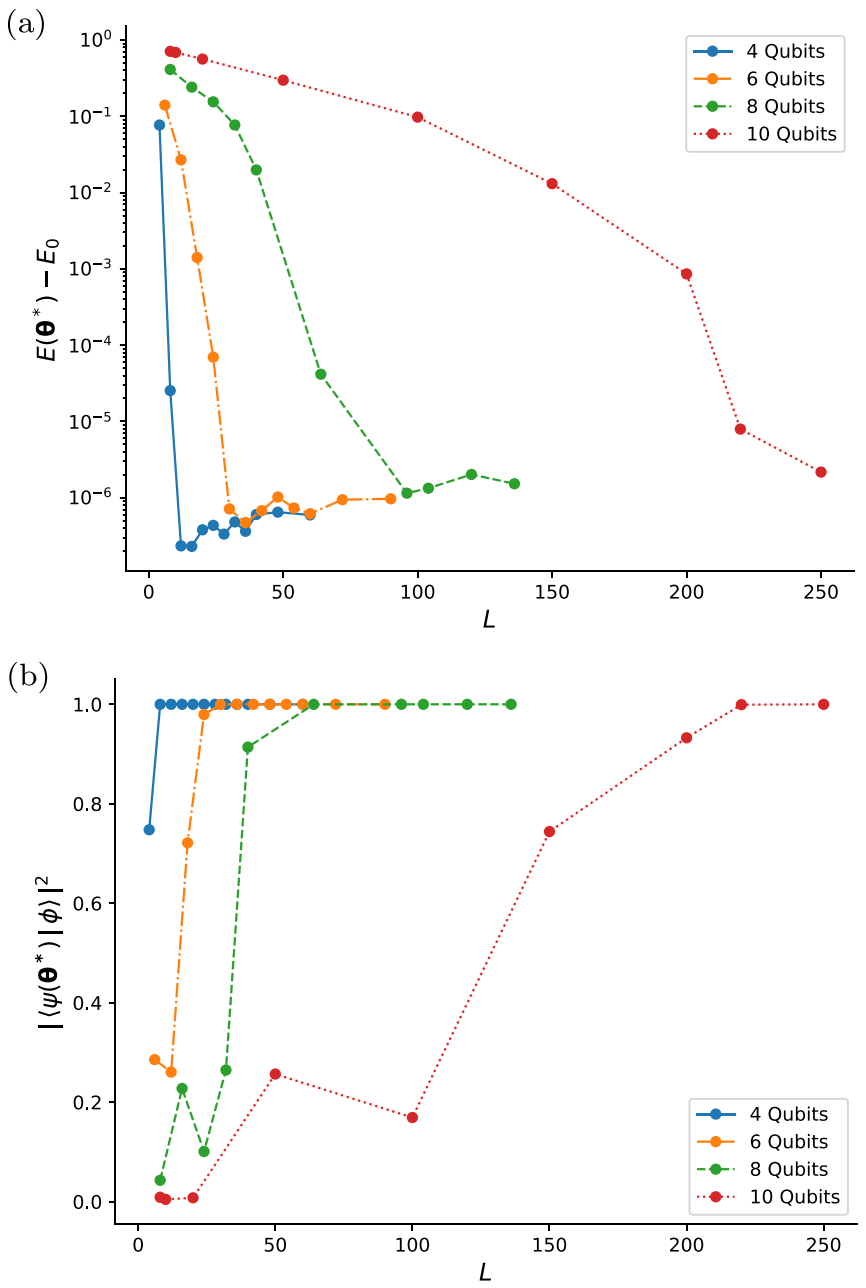

FIG. 10. Single-run VQE outcomes for the SYK model (24) using the layered circuit Ansatz (2) at different depths L. (a) The VQE optimization error $E\left(\boldsymbol{\theta}^{*}\right)-E_{0}$. (b) Fidelity between the optimized and true ground states. As explained near (26) and (27), for the twofold degenerate case $n=4 k+2$, the sum $\left|\left\langle\psi\left(\boldsymbol{\theta}^{*}\right) \mid \phi_{1}\right\rangle\right|^{2}+$ $\left|\left\langle\psi\left(\boldsymbol{\theta}^{*}\right) \mid \phi_{2}\right\rangle\right|^{2}$ is drawn.

adopted the Euclidean distance to improve the convexity of the optimization landscape, such that the closest point $\boldsymbol{\theta}^{*}$ can be found as easily as possible. The Euclidean norm defines trivially a convex landscape. Hence any nonconvexity of the optimization landscape is inherited from the variational circuit itself that fixes how to embed the $n L$-dimensional torus into the Hilbert space.

For an actual estimation of $\varepsilon$, we substitute the Haar unitary integral (29) with the sample mean over $m$ states:

$$
\varepsilon_{m}=\frac{1}{m} \sum_{i=1}^{m} \min _{\boldsymbol{\theta}} \||\psi(\boldsymbol{\theta})\rangle-\left|\phi_{i}\right\rangle \| .
$$

Given a target state $\left|\phi_{i}\right\rangle$, we start with an Ansatz state $|\psi(\boldsymbol{\theta})\rangle$ with $n L$ initial parameters $\boldsymbol{\theta}$, randomly sampled from the uniform distribution $\mathcal{U}(0,2 \pi)^{\otimes n L}$. To reach the optimal parameters $\boldsymbol{\theta}^{*}$ that minimize the distance, we use the Adam optimization algorithm with the hyperparameters (18). Note that the overwhelming majority of Hilbert space is filled with generic states with a high degree of entanglement, so the

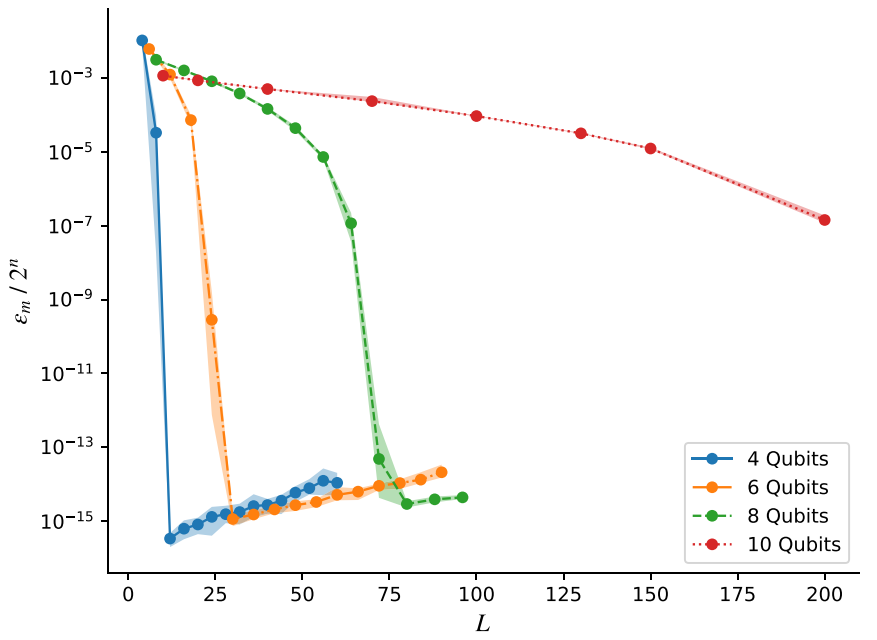

FIG. 11. The parametric (in)expressibility of the layered circuit Ansatz over $m=10$ random target samples, $\varepsilon_{m=10} / 2^{n}$, normalized by the number of state components $2^{n}$. The narrow shaded area denotes the fluctuation across $m=10$ target states.

sample states $\left|\phi_{i}\right\rangle$ will almost never be nongeneric, lowentangling states similar to the Ising ground state.

Figure 11 shows the sample mean $\varepsilon_{m}$ over $m=10$ random target states, divided by the number of state components $2^{n}$. The narrow shaded area displays the fluctuation range of the component-averaged distance $\|\left|\psi\left(\boldsymbol{\theta}^{*}\right)\right\rangle-\left|\phi_{i}\right\rangle \| \cdot 2^{-n}$ across different target states indexed by $i=1, \ldots, 10$. We have selected the minimum distance among the first $\tau \leqslant 500$ optimization steps, which is sufficient since the optimal parameter $\boldsymbol{\theta}^{*}$ is empirically always found in an early stage of the optimization.

We find that the (in)expressibility approaches 0 as the circuit depth $L$ grows. To achieve the high precision

$$
\varepsilon_{m=10} \leqslant 10^{-5} \times 2^{n},
$$

the depth $L$ has to be greater than the threshold values

$$
\begin{array}{ccccc}
n \text { qubits } & 4 & 6 & 8 & 10 \\
\hline L_{\varepsilon} \text { layers } & 10 & 24 & 56 & 150
\end{array}
$$

Note that the slight ramp-up after the steep fall of $\varepsilon_{m=10}$ is caused by the fluctuation of $\boldsymbol{\theta}$ around the optimal point $\boldsymbol{\theta}^{*}$, since the fluctuation size gets amplified for bigger $L$. This is a common phenomenon in gradient-based optimization, where a suitable reduction in the learning rate can turn it into the stable convergence $\boldsymbol{\theta} \rightarrow \boldsymbol{\theta}^{*}$.

In Fig. 11, we have found the following overall trend: Deeper circuits not only are a superset of shallow circuits but also behave more effectively in the gradient-based optimization. Though the functional form of the variational circuit in Fig. 1 is composed of only two types of gates, it can accurately express and reach most quantum states in Hilbert space in the high-depth regime.

\section{DISCUSSION}

From the viewpoint of the vanishing gradient problem, adding more parametric gates to the circuit architecture brings both positive and negative effects on trainability. On the 
negative side, it makes the circuit unitary ensemble closer to quantum 2-design, whose one- and two-point correlators are equivalent to those of Haar random unitaries, so that initial random gradients may decay exponentially with the system size $n$ [4]. On the positive side, however, it enlarges the dimensionality of the parameter space and maintains the norm of random gradients to be finite.

Until now, we have explored one limiting regime, where the impact of exponentially many parameters dominates over the barren plateau phenomenon. It turns out that the highdepth circuit has been very effective in solving the ground states of the Ising and SYK Hamiltonians, as well as in simulating generic random states. One may note a certain degree of qualitative similarity between the VQE optimization trajectory of high-depth circuits, demonstrated in Sec. III B, and the lazy learning [35] in overparametrized neural networks. We speculate that it emerges naturally in any systems involving high-dimensional parameter space. It would be interesting to know why local extrema on the energy landscape of the high-depth circuits can reliably reach a zero VQE error, as studied in Ref. [36] for the neural networks.

Some interesting phenomena that we found during the optimization of low- and intermediate-depth circuits need to be further understood. For low-depth circuits, it is important to characterize what features of the initial points lead to the observed big difference in their minimized energy levels. We are also curious to know what makes the local minima on the energy landscape of intermediate-depth circuits so homogeneous. More generally, we want to understand how the circuit states evolve along a gradient optimization trajectory on average, in terms of various quantum information measures.

We need to concern ourselves with two types of errors for the actual use of variational circuits on near-term quantum devices [37]. Firstly, for accurate estimation of the energy gradient, it is necessary to sample the variational wave function repeatedly, ideally exponentially many times in the system size $n$. Under the assumption that we can collect only a limited number of samples, the energy gradient estimation will inevitably be noisy. Therefore the expressibility and trainability of the variational circuit need to be addressed with noisy gradients, or even without direct gradient computation as in Refs. [38,39]. The high-depth circuit is especially vulnerable to the measurement noise because it contains many parameters and each component of the gradient requires exponentially many measurements for accurate readout, so that the measurement noise can confuse the energy gradient direction. To effectively run it on noisy intermediate-scale quantum (NISQ) devices, it will be essential to apply an appropriate error mitigation technique to reduce the measurement noise and improve output precision [40]. Secondly, and more severely, quantum hardware noise restricts our ability to maintain quantum states when a sequence of layer unitaries acts on them. A noise-induced mechanism that causes the vanishing gradient problem has also been studied in Ref. [41]. Investigations are needed into whether the variational circuit approach can be successful under decoherence of quantum states.

Our PYTHON scripts for the numerical experiments are available [42].

\section{ACKNOWLEDGMENTS}

We thank Boris Hanin and Yaron Oz for helpful discussions. Joonho Kim acknowledges support from National Science Foundation Grant No. PHY-1911298 and the Sivian Fund. The work of D.R. is supported by National Research Foundation of Korea (NRF) Grant No. NRF-2020R1C1C1007591. Our PYTHON scripts for the numerical experiments are written in JAX [43] and QUTIP [44]. The experimental data are managed in WEIGHTS \& BIASES [45].
[1] A. Peruzzo, J. McClean, P. Shadbolt, M.-H. Yung, X.-Q. Zhou, P. J. Love, A. Aspuru-Guzik, and J. L. O'Brien, Nat. Commun. 5, 4213 (2014).

[2] J. R. McClean, J. Romero, R. Babbush, and A. Aspuru-Guzik, New J. Phys. 18, 023023 (2016).

[3] Y. Cao, J. Romero, J. P. Olson, M. Degroote, P. D. Johnson, M. Kieferová, I. D. Kivlichan, T. Menke, B. Peropadre, N. P. D. Sawaya, S. Sim, L. Veis, and A. Aspuru-Guzik, Chem. Rev. 119, 10856 (2019).

[4] J. R. McClean, S. Boixo, V. N. Smelyanskiy, R. Babbush, and H. Neven, Nat. Commun. 9, 4812 (2018).

[5] J.-G. Liu, Y.-H. Zhang, Y. Wan, and L. Wang, Phys. Rev. Res. 1, 023025 (2019).

[6] B. T. Gard, L. Zhu, G. S. Barron, N. J. Mayhall, S. E. Economou, and E. Barnes, npj Quantum Inf. 6, 10 (2020).

[7] K. Seki, T. Shirakawa, and S. Yunoki, Phys. Rev. A 101, 052340 (2020).

[8] R. Wiersema, C. Zhou, Y. de Sereville, J. F. Carrasquilla, Y. B. Kim, and H. Yuen, PRX Quantum 1, 020319 (2020).

[9] E. Grant, L. Wossnig, M. Ostaszewski, and M. Benedetti, Quantum 3, 214 (2019).
[10] G. Verdon, M. Broughton, J. R. McClean, K. J. Sung, R. Babbush, Z. Jiang, H. Neven, and M. Mohseni, arXiv:1907.05415.

[11] M. Cerezo, A. Sone, T. Volkoff, L. Cincio, and P. J. Coles, Nat. Commun. 12, 1791 (2021).

[12] T. Volkoff and P. J. Coles, Quantum Sci. Technol. 6, 025008 (2021).

[13] J. Stokes, J. Izaac, N. Killoran, and G. Carleo, Quantum 4, 269 (2020).

[14] A. Skolik, J. R. McClean, M. Mohseni, P. van der Smagt, and M. Leib, arXiv:2006.14904.

[15] B. Koczor and S. C. Benjamin, arXiv:2008.13774.

[16] S. Sachdev and J. Ye, Phys. Rev. Lett. 70, 3339 (1993).

[17] A. Kitaev, A simple model of quantum holography, Lecture videos, California Institute of Technology, 2015, http://online. kitp.ucsb.edu/online/entangled15/kitaev/ and http://online.kitp. ucsb.edu/online/entangled15/kitaev2/.

[18] J. Maldacena and D. Stanford, Phys. Rev. D 94, 106002 (2016).

[19] B. T. Kiani, S. Lloyd, and R. Maity, arXiv:2001.11897.

[20] M. Belkin, D. Hsu, S. Ma, and S. Mandal, arXiv:1812.11118. 
[21] C. Liu, L. Zhu, and M. Belkin, arXiv:2003.00307.

[22] K. Nakaji and N. Yamamoto, Quantum 5, 434 (2021).

[23] S. Sachdev, Quantum Phase Transitions, 2nd ed. (Cambridge University Press, Cambridge, 2011).

[24] Z. Yang and X. Feng, J. Inequal. Pure Appl. Math. (JIPAM) 3, 12 (2002).

[25] M. Schuld, V. Bergholm, C. Gogolin, J. Izaac, and N. Killoran, Phys. Rev. A 99, 032331 (2019).

[26] D. P. Kingma and J. Ba, arXiv:1412.6980.

[27] L. Wu, Z. Zhu, and W. E, arXiv:1706.10239.

[28] L. Sagun, L. Bottou, and Y. LeCun, arXiv:1611.07476.

[29] P. Chaudhari, A. Choromanska, S. Soatto, Y. LeCun, C. Baldassi, C. Borgs, J. Chayes, L. Sagun, and R. Zecchina, arXiv:1611.01838.

[30] A. M. García-García and J. J. M. Verbaarschot, Phys. Rev. D 94, 126010 (2016).

[31] J. S. Cotler, G. Gur-Ari, M. Hanada, J. Polchinski, P. Saad, S. H. Shenker, D. Stanford, A. Streicher, and M. Tezuka, J. High Energy Phys. 05 (2017) 118.

[32] J. Kim and Y. Oz, arXiv:2102.12534.

[33] A. M. García-García and J. J. M. Verbaarschot, Phys. Rev. D 96, 066012 (2017).
[34] S. Sim, P. D. Johnson, and A. Aspuru-Guzik, Adv. Quantum Technol. 2, 1900070 (2019).

[35] S. S. Du, X. Zhai, B. Poczos, and A. Singh, arXiv:1810.02054.

[36] D. Soudry and Y. Carmon, arXiv:1605.08361.

[37] J. Preskill, Quantum 2, 79 (2018).

[38] N. Maheswaranathan, L. Metz, G. Tucker, D. Choi, and J. SohlDickstein, in Proceedings of the 36th International Conference on Machine Learning Research, Long Beach, California, PMLR Vol. 97 (Curran Associates, Red Hook, NY, 2019), pp. 4264 4273.

[39] S. Welleck and K. Cho, arXiv:2006.03158.

[40] G. S. Barron and C. J. Wood, arXiv:2010.08520.

[41] S. Wang, E. Fontana, M. Cerezo, K. Sharma, A. Sone, L. Cincio, and P. J. Coles, arXiv:2007.14384.

[42] https://github.com/phyjoon/high-depth-vqe.

[43] J. Bradbury, R. Frostig, P. Hawkins, M. J. Johnson, C. Leary, D. Maclaurin, and S. Wanderman-Milne, JAX: Composable transformations of PYTHON+NUMPY programs, 2018, https://github. com/google/jax.

[44] J. R. Johansson, P. Nation, and F. Nori, Comput. Phys. Commun. 184, 1234 (2013).

[45] L. Biewald, Experiment tracking with WEIGHTS \& BIASES, 2020, software available from https://wandb.ai/site. 